\title{
Structural time-dependent damage analysis relying on efficient simulation models and deterioration laws.
}

\author{
L. Ardito
}

Engineer, Engineering Consulting Company, Naples, Italy

N. Basso

Department of Architecture, School of Creative Science and Engineering, Waseda University, Tokyo, Japan

E. Garavaglia

Department of Civil and Environmental Engineering, Politecnico di Milano, Milano, Italy

L. Sgambi

Faculté d'architecture, d'ingénierie architecturale, d'urbanisme, Université Cantholique de Louvain, Tournai

ABSTRACT: Structural time-dependent damage analysis relays on efficient simulation models and deterioration laws. When a probabilistic experiential methodology is applied to existing constructions, the simulation is going to describe the damage state already achieved as well as the further damage evolution. In order to process a reliable damage law, it is necessary to define the deterioration condition in the present and predict it in the future. The parameter law characterisation needs to be identified according to experiential data. This paper presents the parameter characterisation process in a damage law according to non-invasive experiential data collection. The case here discussed is applied to the timber rooftop of a rural structure. Since the structural system is assembled in different periods, which means the life span is multifarious, and the damage degree is different, the calibration procedure reliability and the law versatility can be proved.

This paper is dedicated to Noemi Basso, a friend and esteemed colleague of Waseda University who recently passed away. A flower too delicate to overcome the storms of life.

\section{INTRODUCTION}

The prediction of the degradation behavior over time of structures and their component materials requires the development of simulation models and degradation laws that reliably describe, even if in probabilistic terms, the evolution of this behavior. Degradation laws are reliable when developed on a robust experimental basis. For new constructions the simulation models have a good literature support and can be quite reliable, but the situation becomes more complicated if intervention has to be made on existing structures. The uncertainties involved are not only related to future events, but also to past life. The degradation law, if reliable, should be able to provide both the degradation situation observed today and to foresee its evolution in the coming years. The problem in this case is the estimation of the parameters that characterize the law. They will have to be defined through the experimental data of a targeted diagnostic campaign.

In this paper, the authors present the methodology to calibrate the parameters of a degradation law from some experimental data obtained in a non-invasive diagnostic campaign.
The presented case study is the calibration of the law of degradation applied to wooden roof structures of a rural building, Cascina Cuccagna, in Milan, Italy. The structures are of different ages so the lifetime spent is different as well as the level of degradation reached; this will allow to verify the calibration process and the flexibility of the law considered.

A reliable calibration of the law allows the investigation of the evolution of the degradation in the future years and the planning of maintenance interventions.

\section{THE DEGRADATION LAW AND THE ASSESSMENT OF THE PARAMETERS}

The life-cycle assessment of a building requires the application of a structural model capable of interpreting the evolutionary process of degradation of the structural elements and the materials that compose them. This process is affected by numerous uncertainties due both to the possible variation of the loads to which the building will be subjected and to aggressive attacks from the environment in which it is built.

For these reasons, it is necessary to introduce in the modeling a degradation law able to simulate such events and allow the prediction, in probabilistic terms, of the evolution of performance over the years. (Chow \& Lu 1989, Fujiyama et al. 1995, Thoft-Christensen 2002, Biondini et al. 2008, Gara- 
vaglia et al. 2012, Garavaglia \& Sgambi 2015, Zhou et al. 2015).

Each law is governed by parameters; they are assumed as random variables with a certain probability distribution. The initial value of these parameters has to be defined through quite complex and timeconsuming laboratory tests. Often values present in the literature, and shared by the scientific community, are assumed.

The parameters of the degradation law can be estimated on the basis of some experimental parameters measured in situ with non-destructive and weakly destructive tests. After a correct calibration of the parameters, the law can be introduced in the simulation process of the life-cycle structural behavior.

\subsection{A very adaptable degradation law}

The degradation law proposed in this paper was already applied by the authors, with slight modifications, in the life-cycle assessment of steel, reinforced concrete, and wooden structures (Biondini et al. 2008, Garavaglia et al. 2012, Garavaglia et al. 2014, Garavaglia \& Sgambi 2016, Garavaglia et al. 2018). The law's parameters were deduced by visual inspections or taken from literature. On every occasion, this degradation law has shown a good adaptability to the interpretation of the evolution of degradation.

In this research, the authors want to verify the potentialities of the law in describing the evolution of the degradation of historical woods, if the assessment of the parameters is based on tests carried out on ancient wooden floors and roof trusses.

In structural modeling, the degradation of component elements of the structure can be interpreted as the decrease of the resistant section area of each of them, and consequently the decrease of their resistant capacity:

$$
\begin{aligned}
& A(t)=A_{0}[1-\delta(t)] \\
& \sigma(t)=\sigma_{0}[1-\delta(t)]
\end{aligned}
$$

where: $A(t)$ is the section area that a structural element reaches at time $t$ due to damage, $A_{0}$ is the initial section area of the structural element; $\sigma(t)$ is the residual resistant capacity recorded at time $t$ in the reduced area of the structural element, $\sigma 0$ is the initial resistant capacity of the element and $\delta$ is the damage index describing the degradation value.

The damage index is conceived as follows:

$$
\delta(t)= \begin{cases}\omega^{1-\rho} \tau^{\rho} & , \tau \leq \omega \\ 1-(1-\omega)^{1-\rho}(1-\tau)^{\rho} & , \omega<\tau<1 \\ 1 & , \tau \geq 1\end{cases}
$$

where: $\tau=t / T_{f}$; $t$ is the instant of time; $T_{f}$ is the collapse time, $\rho$ is the parameter that describes the rate of degradation, $\omega$ is the parameter that defines the point of inflection in the distribution and correspond to the trend reversal point of the degradation rate.

The parameters $\omega$ and $\rho$ are assumed to be random variables with assigned probability distribution.

The degradation law (Eq. 3), associated with a structural analysis, will make it possible to predict, in probabilistic terms, the evolution of the degradation of the component elements of a structure and, consequently, the decrease of the structural response over time.

The degradation simulation process can follow the Montecarlo method: as the parameters $\omega$ and $\rho$ change, the degradation law describes, for each structural element, different and variable forms of degradation over time (Fig.1). Therefore, the structural response of the whole system will also be variable over the life of the structure.
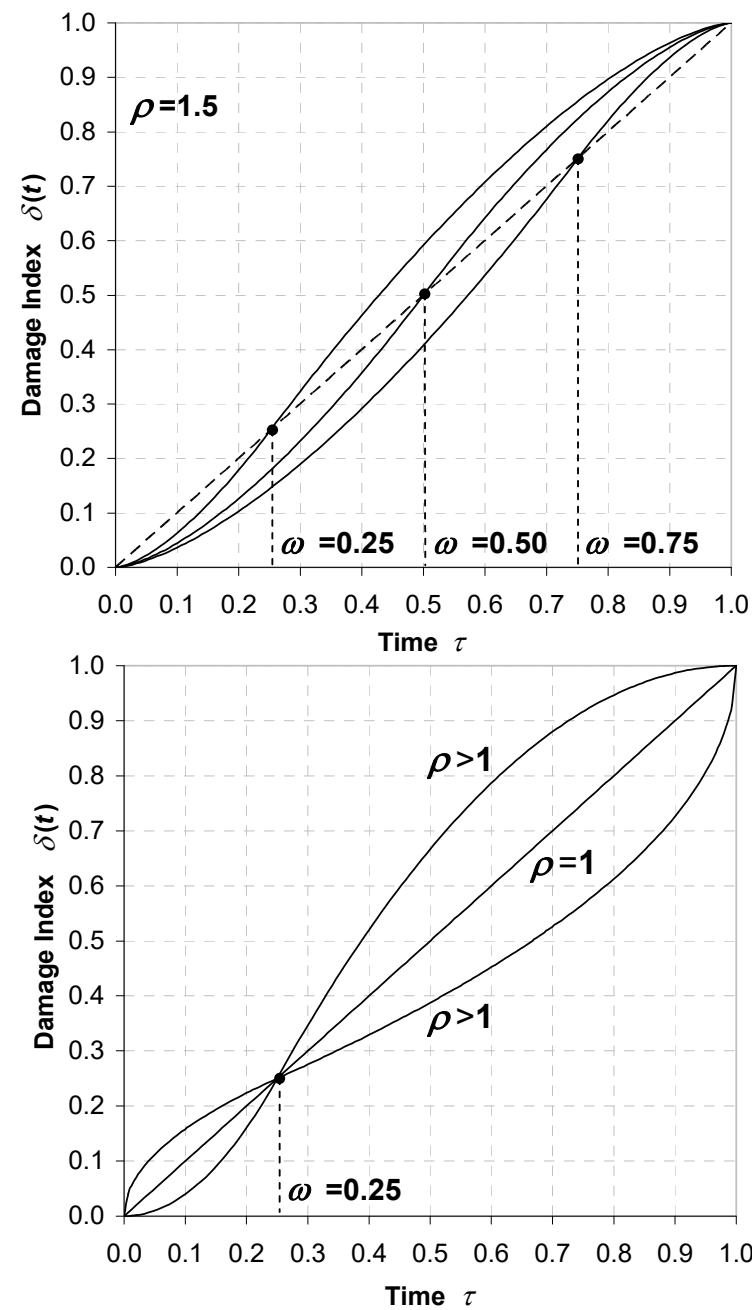

Figure 1. Possible variations in the damage law when parameters $\omega$ and $\rho$ change.

A degradation rate can be associated with both environmental aggression and stress levels $\sigma$ achieved with respect to material strength $\bar{\sigma}$, or $\xi=\sigma / \bar{\sigma}$.

In order to take into account these possible contributions, the parameters $\omega$ and $\rho$ are assumed to be: 


$$
\begin{aligned}
& \rho=\rho_{a}+\left(\rho_{b}-\rho_{a}\right) \xi \\
& \omega=\omega_{a}+\left(\omega_{b}-\omega_{a}\right) \xi
\end{aligned}
$$

where the subscript $a$ refers to the damage associated with environmental aggression while the subscript $b$ refers to damage associated with load variations.

\subsection{Assessment of the residual strength of historical woods by a non-destructive test}

For the calibration of the parameters of the degradation law, an experimental campaign was carried out to evaluate the perforation resistance, performed by the Resistograph $\subset$ device.

These surveys provide punctual and qualitative information on the density variation of the material in the investigated sections.

During the test the instrument records the resistance encountered by the drilling needle providing a graph where on the abscissae is reported the drilling depth (in $\mathrm{cm}$ ) and in ordinate is reported the resistance to penetration expressed in an adimensional percentage scale (Fig. 2,).

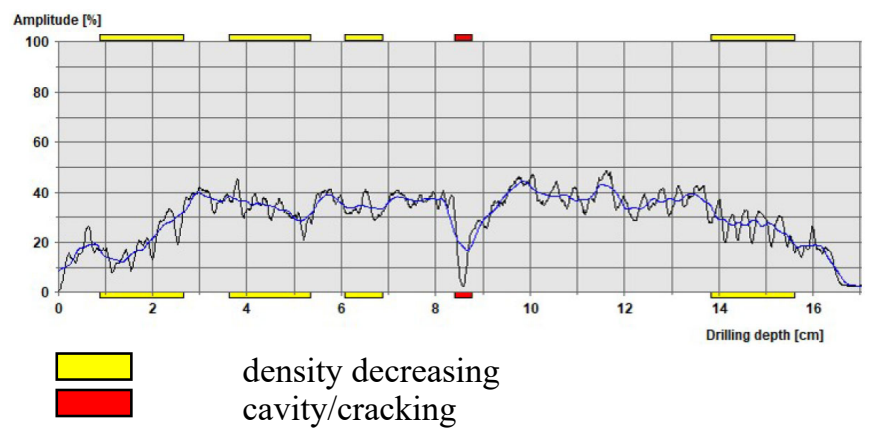

Figure 2. Example of a graph resulting from a test with Resistograph (C)

In a historic building, the decks, ceiling beams and roof elements are often made of wood.

The resistance to perforation is related to the density of the wood and therefore the test allows the identification and measurement of sections where there are variations in density.

Low drilling resistance is synonymous with a decrease in material density and may denote mechanical degradation (checks, ring shakes, etc.) and/or biotic degradation (fungal or xylophagous insect attacks). Peak perforation resistance indicates an increase in density and may indicate the presence of knots.

This type of test provides indications of localized density loss but is unable to estimate the mechanical properties of the material.
2.3 The degradation law for wooden elements: assessment of parameters starting from values measured on site

From the non-destructive tests in situ it is possible to estimate, even if in a qualitative way, the resistance (density) of the elements and the overall level of degradation for the investigated section (Tab. 1).

Table 1. Example of test result with Resistograph $(\mathrm{C}$ on a wooden beam.

\begin{tabular}{lrc}
\hline Parameters measured & results & law parameters \\
\hline Resistance to perforation & $\leq 40 \%$ & $\sigma^{*}$ \\
Density decreasing & $28 \%$ & \\
Cavity and/or cracking & $11 \%$ & \\
Overall degradation & $38 \%$ & $\delta^{*}$ \\
\hline \hline
\end{tabular}

Knowing the period of construction of the building and the results of the tests performed on site, it is possible to estimate the level of damage $\delta^{*}$ achieved at the time $t^{*}$ (today). As a damage parameter $\delta^{*}$ can be assumed the overall degradation percentage normalized to 1 .

Considered $T_{f}$ as the survival limit established by the regulation (e.g. 475 years) it is possible to refer to the normalized instant, $\tau^{*}$, through the ratio:

$$
\tau^{*}=\frac{t^{*}}{T_{f}}
$$

Knowing the instant $\tau^{*}$ and the normalized percentage damage $\delta^{*}$, it is also possible to assess the parameter $\rho^{*}$, which describes the degradation rate:

$$
\rho^{*}=\frac{\delta^{*}}{\tau^{*}}
$$

The parameter $\omega^{*}$ is then defined by the ratio:

$$
\omega^{*}=\left[\frac{\delta^{*}}{\left(\tau^{*}\right)^{\rho^{*}}}\right]^{\frac{1}{\left(1-\rho^{*}\right)}}
$$

Using the on-site analysis of the structure's woods, an average value for the parameters $\rho^{*}$ and $\omega^{*}$, and their standard deviations, can be derived.

For parameters $\rho_{\mathrm{a}}$ and $\rho \mathrm{b}, \omega_{\mathrm{a}}$ and $\omega \mathrm{b}$, defined by Eq. (4) and (5), the authors assume that the influence of the environment on degradation corresponds to $20 \%$ of the degradation recorded, while mechanical and load variations contribute to the remaining $80 \%$ :

$$
\begin{array}{ll}
\rho_{a}=\left(\rho^{*} \cdot 0.2\right) ; & \rho_{b}=\left(\rho^{*} \cdot 0.8\right) \\
\omega_{a}=\left(\omega^{*} \cdot 0.2\right) ; & \omega_{b}=\left(\omega^{*} \cdot 0.8\right)
\end{array}
$$

These parameters will provide the basis for the Monte Carlo simulation of the behavior of structural elements over time. The purpose of this simulation 
will lead to identify a probable moment of failure in order to schedule appropriate maintenance scenarios.

\section{THE CASE STUDY: CASCINA CUCCAGNA IN MILAN (ITALY)}

In 2017 the Politecnico di Milano and the Associazione Consorzio Cascina Cuccagna (ACCC) have obtained funding from Fondazione Cariplo for the static and seismic adequacy of Cascina Cuccagna (Fig. 3). The funded project is the PRE.CU.R.S.OR

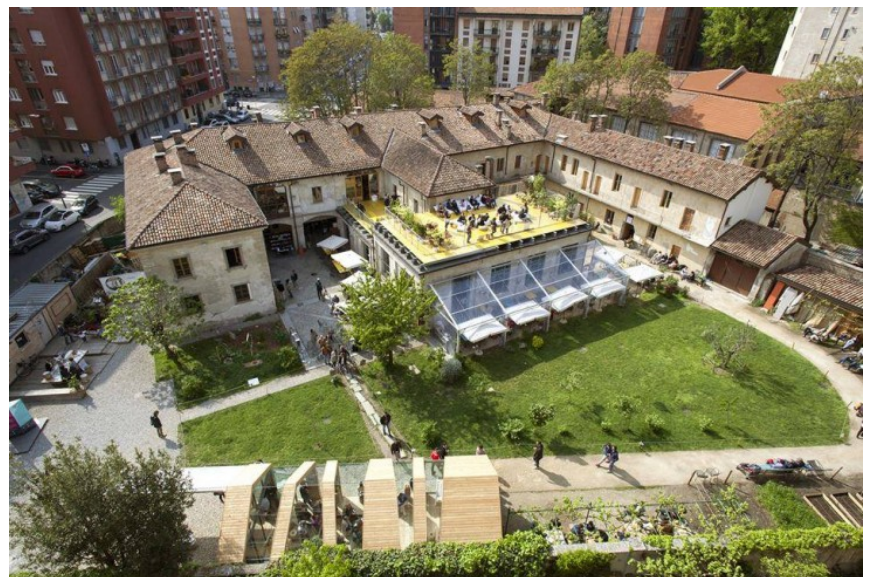

Figure 3. Cascina Cuccagna: aerial view (kindly offered by $\mathrm{ACCC})$

Cascina Cuccagna is a typical rural structure in the Lombard countryside, protected by the Cultural Heritage. The building requires maintenance and static and seismic adequacy.

The PRE.CU.R.S.OR project aims to define lines of intervention for the maintenance project that are repeatable to other historical buildings of a similar type.

The project required a first phase of investigation into the state of degradation of structural elements and the presence of specific vulnerabilities. It is in this phase that the calibration of the degradation law presented could be carried out.

The penetration resistance tests were carried out on most of the woods of the Cascina in order to have a reliable and sufficient data set to represent a statistical sample.

The woods on which the tests have been performed were woods of different ages and this has allowed the evaluation of the adaptability of the degradation law (3) to the variation of the resistance and degradation detected and to the variation of the construction period (Tab. 2 and Fig. 4).
Table 2. Experimental parameters obtained by eq. (6), (7) and (8) and used in (9) and (10).

\begin{tabular}{llllrr}
\hline $\begin{array}{l}\text { Year of } \\
\text { Construction }\end{array}$ & $\begin{array}{c}t^{*} \\
\text { (to 2018) }\end{array} \tau^{*}$ & $\delta^{*}$ & $\rho^{*}$ & $\omega *$ \\
\hline 1722 & 296 & 0.6230 .31 & 0.05 & 0.155 \\
1857 & 161 & 0.3390 .24 & 0.71 & 0.104 \\
1887 & 131 & 0.2760 .27 & 0.98 & 0.100 \\
1893 & 125 & 0.2630 .26 & 1.01 & 0.097 \\
1897 & 121 & 0.2550 .30 & 1.18 & 0.101 \\
\hline
\end{tabular}

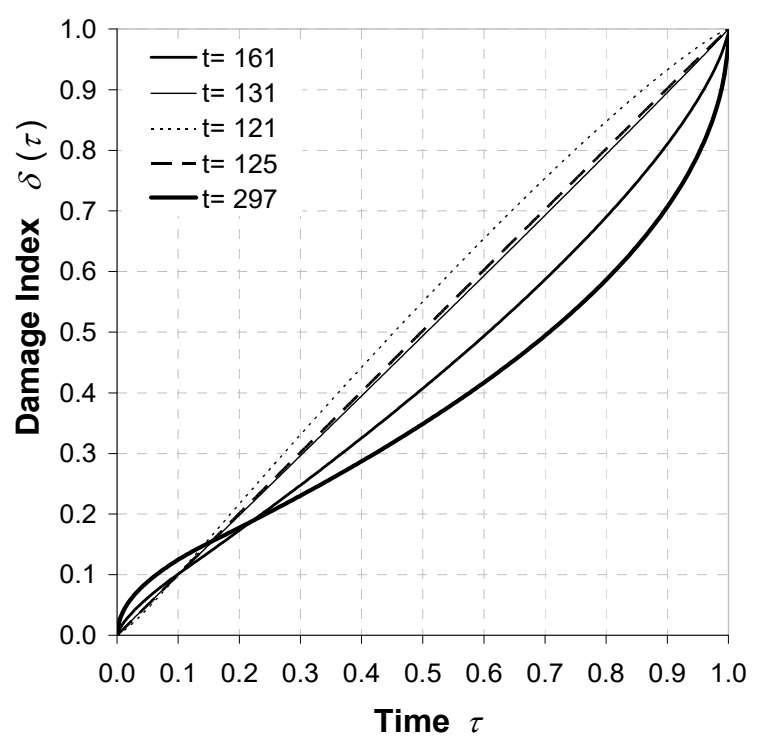

Figure 4. Damage curves defined for the lifetime, $t$, characteristic of the wooden elements of the Cascina Cuccagna, and derived from parameters (9) and (10) detected with the experimental tests and included in the Eq. (4) and (5).

\subsection{Application of the model to a roof truss of the Cascina Cuccagna}

The degradation law (3), whose parameters have been estimated on the basis of experimental tests performed on site, is applied to a roof truss of the Cascina Cuccagna.

The roof truss under investigation is shown in Figure 5.

It was made of chestnut wood, built in 1887 and always kept in a closed rural environment.

The static scheme used is shown in Figure 6. The loads are inclusive of dead loads and live loads weighed with corrective coefficients as required by Italian regulations for the analysis at the ultimate limit states. The vertical element has not been considered because its static purpose is only to constrain the inclined elements. 


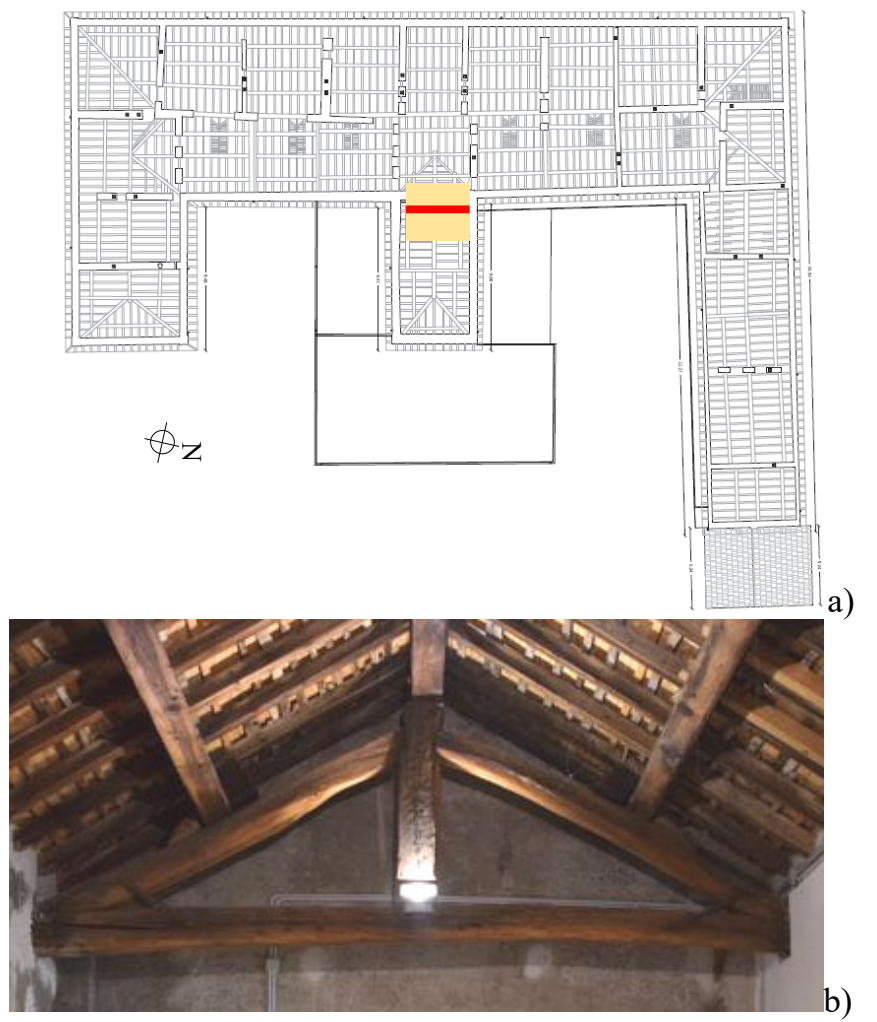

Figure 5. Cascina Cuccagna: (a) plan of the building roof; in red the truss analyzed; in yellow the load area. (b) The roof wood truss (Authors property).

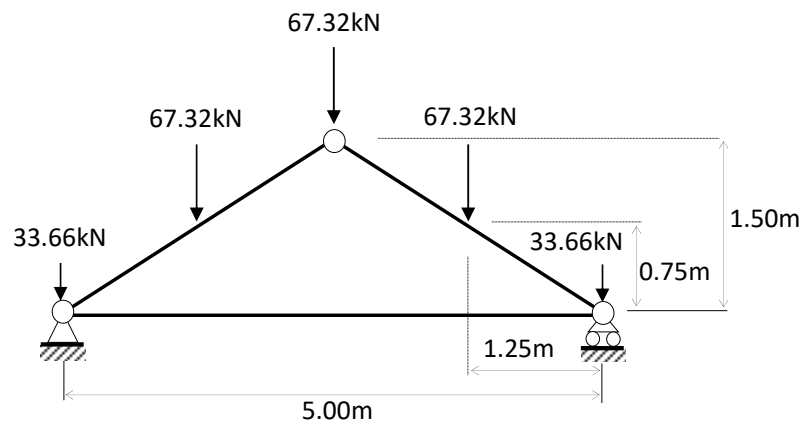

Figure 6. Static scheme of the wooden roof truss

\subsection{Monte Carlo approach}

In order to investigate the life-cycle of the structure during the building service life along with the damages affecting it, a Monte Carlo simulation implemented with the damage law (3) is run. Damage parameters $\rho_{a}, \rho_{b}, \omega_{a}, \omega_{b}$, obtained by Eq. (9) and (10) and failure time $T_{f}$ are modelled as random variables with assigned probability distributions: Normal distribution for parameters $\rho$ and $\omega$; Gamma distribution for time $T_{f}$. The initial values of these parameters were the values shown in Table 2 with standard deviation obtained by the experimental values statistical elboration.

Using RDM.m random choice function of MatLab code (Matworths Inc. 2005), 1000 simulations have been performed. At each run, a random value, extracts from the assigned probability function, is linked to each variable, and implemented in the law (3). Then the deterioration law is applied to the time-dependent structural analysis in order to define the structural response in terms of decrease in stiffness member by member and failure time for the whole structure.

This procedure, implemented with the damage law (3), can provide important data: a variety of failure time samples for the evaluation of the average failure time $T_{f}$ and the estimation of their probability density function $F_{\text {failure }}(t)$ (Fig. 7 ).

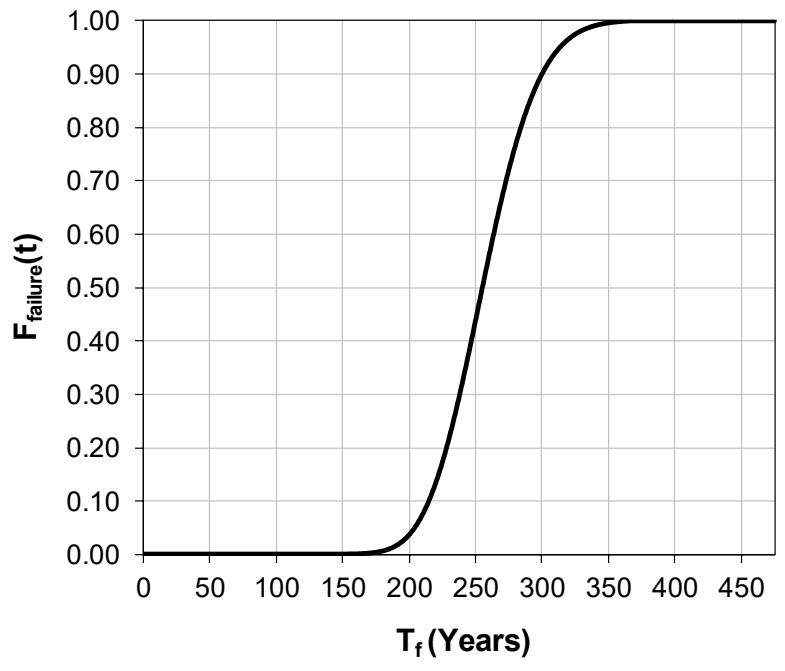

Figure 7. Roof Truss: cumulative distribution function of structural failure time.

The cumulative distribution function shows how the probability of collapse for a chestnut wood structure, built around 1887 and maintained in a closed rural environment, has a first increase in probability of collapse starting from the age of 215 years (probability $10 \%$ ) with a more rapid increase in the following years (at 247 years the probability rises to $47 \%$ ).

It is therefore important to define limits of reliability, or safety margins, and the calculation of the relative probabilities of achievement.

\subsection{Reliability function.}

Reliability $R(t)$ describes the performance of a system over time, and is defined as the probability that the system does not fail by time $t$ (e.g. Evans 1992). This definition is extended here, denoting by $\bar{R}(t)$ the probability that a system will not exceed a given safety margins by time $t$ (Garavaglia et al. 2016). Reliability can be then quantified by the random variable $\bar{T}$, defined as the time needed to reach, or exceed, the safety margins defined.

Thus, from this point of view, the reliability function is given by:

$$
\bar{R}(t)=\operatorname{Pr}(\bar{T}>t)=1-F_{\bar{T}}(t)
$$


here $F_{\bar{T}}(t)$ is the cumulative distribution function of $\bar{T}$.

Figure 8 shows the reliability function related to the roof truss analyzed. It shows how the achievement of moderate but already significant damage from a structural point of view could occur in the next 10 years.

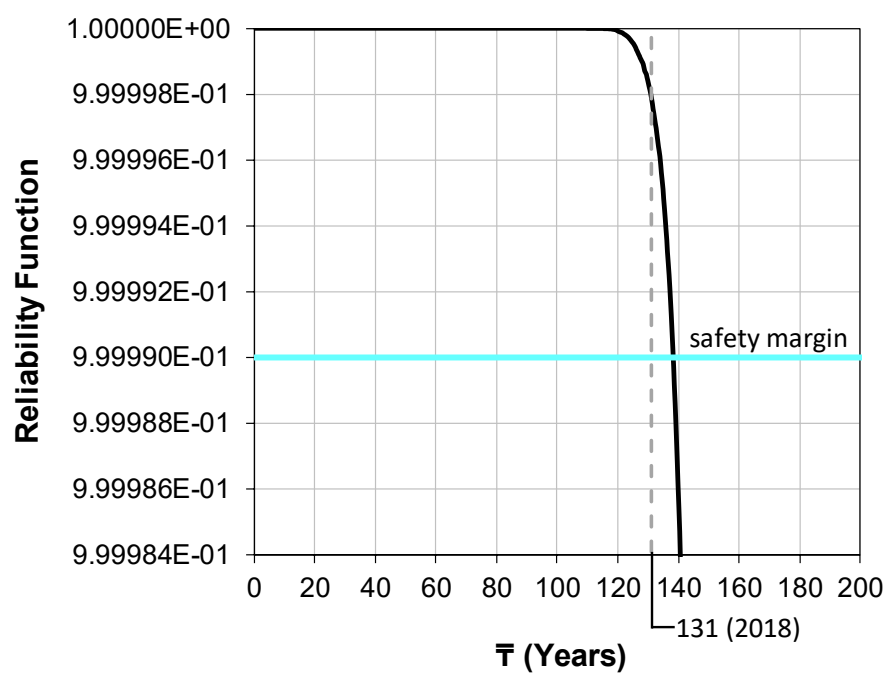

Fig. 8 Reliability function, in evidence the probable time in which the safety margin chosen could be reached.

The study of the reliability function allows the scheduling of maintenance interventions that can keep the structure safe and efficient over a longer life span.

\section{RESULTS AND COMMENTS}

Based on the presented results, we can conclude that the roof truss analyzed, while showing a reduction in drilling resistance of around $38 \%$, still has good load-bearing capacity. It is, however, prudent to intervene with a more careful investigation of the type of biological degradation present and possible rottenness, as well as to plan a maintenance action that can restore the roof truss to a resistant capacity higher than the current one.

With maintenance programming, the lifetime of the structure is certainly extended and its performance guaranteed in safety.

Figure 9 shows an example of a maintenance plan built based on the reliability analysis carried out.

With each maintenance operation, it will be difficult for the structure to recover all the performance lost, so the intervals between a maintenance operation and the next one may be shorter from time to time.

Assuming that immediate maintenance is carried out and constant maintenance is planned, it is possible to maintain the structure at excellent performance levels still for a long time (Fig. 9).

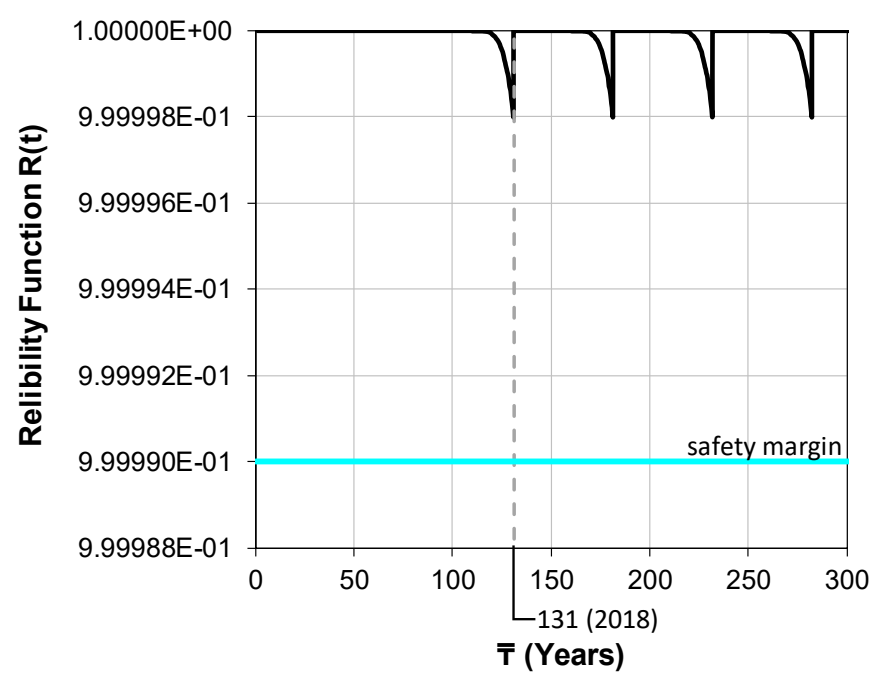

Fig. 9 Possible maintenance planning for the roof truss structure analized.

\section{CONCLUSIONS}

This paper once again demonstrates how a prediction tool, even if in probabilistic terms, of the performance evolution of a structural element during its lifecycle is fundamental to plan an adequate maintenance scenario that can preserve the element at good levels of efficiency.

This approach is applied both in the design phase of new construction elements and in the investigation of the performance of existing structures. In both cases, an important uncertainty is the degradation that could affect the element in design or that has attacked the existing element.

In the literature this uncertainty is addressed through the development of damage laws able to interpret, in probabilistic view, the evolution of the possible degradation to which the structure could be subject.

The emerging question is the calibration of the parameters inherent in these laws, which is even more important when dealing with the performance evaluation of an existing structure.

In this paper is presented an adaptable degradation law for which the calibration of the parameters has been carried out starting from experimental results obtained on site.

The application of this degradation law to the evaluation of the residual performance of a wooden roof truss, built in 1887, has made it possible to verify that the law is able to describe the degradation achieved by the truss today, but also to predict its future evolution.

The Monte Carlo simulation, implemented with the degradation law and parameters calibrated on experimental data, was applied to the case study. The results of this simulation showed that the performance of the roof truss is still good, however, a planned maintenance action could help to extend the 
life of the structure and the building of which it is part.

\section{Founding}

The following research is funded by the CARIPLO Foundation, Milan, Italy, in the context of the 2017 Art and Culture-Cultural Heritage at Risk Calls, Project PRE.CU.R.S.OR.

\section{Acknowledgment}

Thanks to the ACCC association of Cascina Cuccagna, the design team of Hydea S.p.A. for the collaboration undertaken in this project.

\section{REFERENCES}

Biondini F., Frangopol D.M., \& Garavaglia E., 2008. Damage Modeling and Life-Cycle Reliability Analysis of Aging Bridges. Proc. of IABMAS'08 4th Int. Conf. on Bridge Maintenance, Safety and Management, Seoul, Korea, 13-17 July, Koh H-M \& Frangopol D.M. (Eds.), 2008, Taylor \& Francis Groups, London, 2229-2236.

Chow, C.L. \& Lu, T.J. 1989, On evolution laws of anisotropic damage, Engineering Fracture Mechanics 34(3), \&79-701.

Evans, D.H., (1992). Probability and its Applications for Engineers. Marcel Dekker Inc., New York, NJ, USA.

Fujiyama, K., Murakami, I., Yoshioka, Y. Okabe, N., 1995. Methods of predicting deterioration and damage of structural member and prediction apparatus for use with method. United States Patent 5455777.

Garavaglia E., \& Sgambi L. 2015. The use of a credibility index in the life-cycle assessment of structures, Structures and Infrastructures, 11(5), 683-694.

Garavaglia E., \& Sgambi L., 2016. Selective maintenance planning of steel truss bridge based on the Markovian approach, Engineering Structures, 125 (15 oct. 2016), 532545 ,

Garavaglia E., Basso N., \& Sgambi L., 2018. Probabilistic lifecycle assessment and rehabilitation strategies for deteriorating structures: a case study. International Journal of Architectural Heritage, 12(6), 981-996,

Garavaglia E., Sgambi L., \& Basso N. 2012 Selective maintenance strategies applied to a bridge deteriorating steel truss, Proc. of IABMAS12 6th Int. Conf. on Bridge Maintenance, Safety and Management, Lake Como, Italy 08-12-July, Biondini F. \& Frangopol D.M. (Eds)., Taylor \& Francis Group, London, $1764-1770$,

Garavaglia E., Sgambi L., \& Basso N. 2014 Markovian approach for life-cycle assessment and maintenance planning of a steel bridge Proc. of IALCCE2014, 4th Int. International Symposium on Life-Cycle Civil Engineering, LifeCycle of Structural Systems Design, Assessment, Maintenance and Management, Tokyo, Japan, 16-19 November, Furuta H., Frangopol D.M., and Akiyama M. (Eds), CRC Press, 582-588.

Thoft-Christensen P. 2002. Deterioration of concrete structures, Pro. IABMAS 2002, First International Conference on Bridge Maintenance, Safety and Management, Barcellona, Spain, 14-17 June 2002. Aalborg: Dept. of Building Technology and Structural Engineering. Structural Reliability Theory, No. 204, Vol. R0130.

Zhou, Y., Tian, H., Sui L., Xing, F., and Han, N. 2015. Strength deterioration of concrete in sulfate environment: an experimental study and theoretical modelling, Advance in Materials Science and Engineering, Vol. 2015, Art. ID 951209, 1-12. 